\title{
Equilibrium physics breakdown reveals the active nature of red blood cell flickering
}

\author{
H. Turlier ${ }^{1,2,3}$, D. A. Fedosov 4 , B. Audoly ${ }^{3,5}$, T. Auth ${ }^{4}$, N. S. Gov 6 , C. Sykes ${ }^{2}$, \\ J.-F. Joanny ${ }^{2,7}$, G. Gompper ${ }^{4}$, T. Betz ${ }^{2,8 *}$
}

\begin{abstract}
Affiliations
${ }^{1}$ European Molecular Biology Laboratory, Meyerhofstrasse 1, 69117 Heidelberg, Germany.

2 Institut Curie, PSL Research University, CNRS, UMR 168, F-75005, Paris, France.

${ }^{3}$ Sorbonne Universités, UPMC Université Paris 06, Paris, France

${ }^{4}$ Institute of Complex Systems and Institute for Advanced Simulation (ICS-2/IAS-2),

Forschungszentrum Jülich, 52425 Jülich, Germany.

${ }^{5}$ CNRS, Institut Jean Le Rond d'Alembert UMR7190, 4 place Jussieu, Paris, France.

${ }^{6}$ Dept. of Chemical Physics, Weizmann Institute of Science, 76100 Rehovot, Israel.

${ }^{7}$ ESPCI-ParisTech, 10 rue Vauquelin, 75005 Paris, France.

${ }^{8}$ Institute of Cell Biology, Center for Molecular Biology of Inflammation, Cells-in-Motion Cluster of Excellence, Münster University, Von-Esmarch-Strasse 56, D-48149 Münster, Germany;
\end{abstract}

*Correspondence to: timo.betz@uni-muenster.de

\begin{abstract}
Red blood cells, or erythrocytes, are seen to flicker under optical microscopy, a phenomenon initially described as thermal fluctuations of the cell membrane. But recent studies have suggested the involvement of non-equilibrium processes, without definitively ruling out equilibrium interpretations. Using active and passive microrheology to directly compare the membrane response and fluctuations on single erythrocytes, we report here a violation of the fluctuation-dissipation relation, which is a direct demonstration of the non-equilibrium nature of flickering. With an analytical model of the composite erythrocyte membrane and realistic stochastic simulations, we show that several molecular mechanisms may explain the active fluctuations, and we predict their kinetics. We demonstrate that tangential metabolic activity in the network formed by spectrin, can generate curvature-mediated active membrane motions. We also show that other active membrane processes represented by direct normal force dipoles may explain the observed membrane activity. Our findings provide solid experimental and theoretical frameworks for future investigations of the origin and function of active motion in cells.
\end{abstract}




\section{Main Text}

One of the first observations of red blood cells (RBC) by optical microscopy during the nineteenth century was the 'vibrating' fluctuation of its plasma membrane ${ }^{1}$. Important biological functions of this flickering have been suggested, such as decreased interactions between cells ${ }^{2}$ or facilitated diffusion of membrane proteins ${ }^{3}$. Today, flickering analysis is envisaged as a promising tool for high-rate malaria diagnosis ${ }^{4}$. However, although equilibrium fluctuation models ${ }^{5-7}$ have been used to infer mechanical properties of RBCs since the seminal work of Brochard \& Lennon ${ }^{8}$, the fundamental question whether membrane fluctuations are driven by an active process or simply by thermal agitation remains controversial ${ }^{9-12}$.

Possible active metabolic origins were conjectured more than 60 years ago ${ }^{13}$, but were readily questioned by the observation that flickering arises in reconstituted membranes as well. By comparing membrane fluctuations directly between healthy RBCs and cells depleted of ATP (adenosine triphosphate), recent experiments find a decrease of fluctuations upon ATP-depletion, hence suggesting that an active process may contribute to membrane undulations ${ }^{6,14-16}$. However, the passive mechanical properties of the RBC membrane themselves depend strongly on ATP, as illustrated by the stiffening of the RBC membrane on starvation ${ }^{17-19,6,15}$, that correlates with a sudden transition from discocyte to spiculated echinocyte shapes ${ }^{20,21}$. Therefore a direct and compelling explanation for the decrease of fluctuations observed in ATP-depleted cells is the stiffening of the membrane ${ }^{22,23}$ rather than the loss of putative active (that is, non-equilibrium) fluctuations. To make a different assessment of the involvement of metabolic activity in flickering, an attractive approach was used by Tuvia et $\mathrm{al}^{14}$ : at equilibrium, the mean-square fluctuations amplitude is a thermodynamic variable, which, by definition, cannot depend on the dynamics, and in particular on medium viscosity. Tuvia et $\mathrm{al}^{14}$ reported a viscosity dependence of the mean-square fluctuation amplitude in a frequency range of $0.3-30 \mathrm{~Hz}$ on changing the suspending medium and therefore drew the conclusion of a metabolic origin of flickering. However, recent analysis of fluctuations over a larger bandwidth $(0.1-1000 \mathrm{~Hz})$ shows no detectable dependence of fluctuation amplitudes on medium viscosity ${ }^{6}$, as also confirmed here (Supplementary Figure S1 and SI Appendix C), hence making the conclusions of Tuvia et al equivocal. Recent publications ${ }^{15,24}$ reported non-Gaussian features in the RBC membrane fluctuations distribution and associated them to an active mechanism. However, an equilibrium fluctuations distribution is also likely to exhibit non-Gaussian tails, as soon as anharmonicities are present in the membrane ${ }^{25,26}$. On the other hand, the fluctuations distribution of a nonequilibrium system can be perfectly Gaussian ${ }^{25,27}$. As a consequence, the observation of Gaussian fluctuation distributions $6,9,11,12$ does not necessarily imply that RBC flickering is purely thermal. ATP-dependent temporal correlations, consistent with an active component, were recently identified $^{16}$, however their interpretation is model-dependent and does not provide unequivocal evidence for active forces.

An unquestionable hallmark of equilibrium systems is alternatively found in the fluctuationdissipation theorem (FDT), which relates directly mechanical properties to fluctuations in the linear regime ${ }^{28}$. By measuring the response function and spontaneous fluctuations for the same system and for the same experimental conditions, it is possible to assert directly the presence of 
non-equilibrium processes, as recently demonstrated in vitro for hair bundles ${ }^{29}$, active gels ${ }^{30}$ and suspended bone cells ${ }^{31}$. A series of techniques allow the measurement of cellular mechanics ${ }^{32}$, and the RBC membrane response was measured previously using micropipette aspiration ${ }^{33}$, electric fields ${ }^{34}$, or optical tweezers ${ }^{10,35}$, but never compared directly to the fluctuations in the same cell. Here, we use active and passive microrheology to measure directly single erythrocyte membrane response and fluctuations for the same cell and over timescales from milliseconds up to tens of seconds, a range which remains unattained so far ${ }^{10}$.

The experimental approach relies on a time-shared multiplexed optical tweezer, generating four independent traps that control the position of four streptavidin-functionalized polystyrene beads attached to a biotinylated RBC at opposite sites along its rim (figure 1, Supplementary figure S2). The beads allow us to apply well-defined forces on the cell membrane, but they can also track local membrane shape deformation with sub nm-accuracy and avoid problems related to the convolution between the cell edge and the detection laser when directly tracking the membrane ${ }^{6,36}$. Using this setup, we can detect both the free fluctuations and mechanical response of the cell membrane with sub-millisecond temporal resolution, thanks to an interferometric detection system (figure 1a). Three beads (figure 1a inset) serve as handles to hold the cell $20 \mu \mathrm{m}$ above the coverslip surface in the observation chamber. The fourth bead is used as a probe (figure 1a inset) to detect free fluctuations, or to measure the mechanical response of the membrane under external driving.

In a first experimental step, the free membrane fluctuations are determined by reducing the laser trap power on the probe bead from $5 \mathrm{~mW}$ to $0.1 \mathrm{~mW}$. This value is insufficient for stable trapping but it allows laser-tracking of the bead position. We checked carefully that the presence of this weak trap does not restrict the fluctuations (Supplementary figure S3). Therefore, the bead motion reflects directly membrane fluctuations (figure $1 \mathrm{~b}$ ), which are characterized by their power-spectrum-density (PSD) $C(f)$ expressed as function of the frequency $f$. Furthermore, the influence of the bead on the PSD remains furthermore lower than measurement error in the frequency range probed here when compared to the free membrane (Supplementary figure S4). Immediately after PSD measurement, the mechanical response is determined for the same cell in a second experiment, where the three handle traps remain unchanged but the average laser power on the probe bead is increased to $5 \mathrm{~mW}$. Sinusoidal oscillations of the probe trap at a driving frequency $f$ (figure 1c, blue) displace it over a $200 \mathrm{~nm}$ amplitude (figure 1c, green). The complex response function $\chi(f)$ is calculated as the ratio between the Fourier transforms of the position $x(f)$ and force $F(f)$ at the driving frequency $f$. The real and imaginary parts of the response function, $\chi^{\prime}$ and $\chi^{\prime \prime}$, correspond respectively to the elastic and the dissipative components of the linear response of the RBC membrane to the applied force. To obtain the frequencydependent response function, we repeat this active rheology measurement for driving frequencies ranging from $0.2 \mathrm{~Hz}$ to $1 \mathrm{kHz}$. We check the linearity of the response by investigating the forcedeformation relation as well as the absence of higher harmonics in the bead displacement and response spectra for each driving force (Supplementary figure S5). For both active and passive 
microrheology measurements, the handle beads move around their equilibrium position. Hence, the probe bead movement combines the mechanics of the cell and the handle traps. Fluctuations and mechanical response function are measured under the exact same experimental conditions, which enables us to directly test the validity of the $\mathrm{FDT}^{28}$, which reads

$$
C(f)=2 k_{B} T \chi^{\prime \prime}(f) /(2 \pi f)
$$

where $k_{B} T$ is the thermal energy, with $k_{B}$ being Boltzmann's constant.

We check for violation of the FDT in fresh RBCs, as well as in cells that were ATP-depleted by incubation in glucose-free medium (GFM) at $37^{\circ} \mathrm{C}$ for $16 \mathrm{~h}$ (partial ATP-depletion) and $>48 \mathrm{~h}$ (full ATP-depletion). For fully ATP-depleted cells (nine experiments on seven cells) figure 2a displays the dissipative part of the response function and the predictions from the FDT based on the PSD measurements (equation (1)). The observed collapse of both data sets demonstrates the validity of the FDT for a purely passive RBC, as expected. In strong contrast, fresh RBCs (21 experiments on 9 cells) show a clear violation of the FDT in the low frequency regime $f<10 \mathrm{~Hz}$ (figure 2b). This result is a direct and conclusive demonstration that an active mechanical process contributes to the flickering of the RBC membrane at timescales above $100 \mathrm{~ms}$. Conversely, the collapse of free fluctuations and response at high frequencies ( $\mathrm{f}>10 \mathrm{~Hz}$ ) indicates the validity of the FDT for rapid fluctuations. Therefore, thermal agitation is the dominant source of fluctuations at short timescales $(<100 \mathrm{~ms})$. We conclude that fluctuation measurements can be reliably used to infer mechanical properties of the RBC membrane only in this high frequency limit. The validity of previous studies, which used equilibrium theories to extract mechanical information on the RBC membrane, has therefore to be critically checked. The comparison of the dissipative response for the three conditions (zero, partial and full ATP depletion) in figure 2c confirms the strong metabolic dependence of passive membrane properties noted previously $6,12,18,22,23$.

To measure directly the metabolic energy input which drives active fluctuations, we use the concept of effective energy ${ }^{6} E_{\text {eff }}(f)$, defined as the thermal energy at a frequency $f$ that would be necessary to recover the FDT in the non-equilibrium system $E_{\text {eff }}(f)=C(f) \pi f / \chi^{\prime \prime}(f)$. The metabolic energy input driving active flickering is visualized directly above $1 k_{\mathrm{B}} T$ in figure $2 \mathrm{~d}$. Interestingly, ATP-depletion of RBCs is not fully complete after $16 \mathrm{~h}$ of incubation in glucosefree medium ${ }^{17,37}$. The effective energy coincides with the thermal energy at high frequency for all conditions, but it increases up to $23 \mathrm{k}_{\mathrm{B}} \mathrm{T}$ at low frequencies for fresh $\mathrm{RBCs}\left(\mathrm{ref}^{27}\right)$. Integrating this deviation over the full spectrum gives an estimate of the power $P_{f} \sim 0.025 \mathrm{fW}$ used for driving the membrane fluctuations. This is only a very small fraction of the total metabolic energy turnover in the RBC $\left(\mathrm{ref}^{38}\right)$ of $P_{\mathrm{RBC}} \sim 10 \mathrm{fW}$.

Diverse metabolic processes have been identified in the RBC membrane. We use here analytical and simulation models to explore their relevance in explaining the observed active fluctuations. 
The membrane consists of a network of spectrin filaments anchored to a lipid bilayer, as illustrated in figure 3a. Biochemists identified several phosphorylation sites directly in the network: at the transmembrane protein complexes $4.1 \mathrm{R}\left(\operatorname{refs}^{23,39,40}\right)$ and ankyrin ${ }^{41}$, which anchor the network in the bilayer, but also at the spectrin chains ${ }^{22}$. These phosphorylations have been associated with a decreased mechanical strength of the membrane ${ }^{42,23,43,6,15}$, but the underlying molecular mechanism remains unclear. Instead of considering one specific process, we argue here that any relevant spectrin phosphorylation should lead to a local decrease of the network shear modulus, as this single parameter characterizes its mechanical properties. Our mean-field approach encompasses therefore the filament detachment hypothesis ${ }^{43,44}$, but also other potential spectrin metabolic processes. More precisely, we assume a simple two-state dynamics, where phosphorylation sites can switch between active and inactive states with transition rates $k_{a}$ and $k_{i}$ respectively, defining hence a mean activity

$$
\left\langle n_{a}\right\rangle=\frac{k_{a}}{k_{a}+k_{i}} \text {. The network shear modulus }
$$

fluctuates accordingly around a mean value $\langle\mu\rangle=\mu_{0}\left(1-\left\langle n_{a}\right\rangle\right)$, that decreases with phosphorylation. Modeling the RBC membrane mechanics remains a challenging task, as multiple length and timescales are involved. Most previous composite models are hence numerical $^{45-48}$ or limited to flat geometries ${ }^{43,44,49-51,16}$. We derive here an active composite membrane model in spherical geometry, while considering explicitly the finite elasticity of the spectrin network and its sliding relative to the bilayer.

The spectrin cytoskeleton is idealized as a perfect triangular network of springs. In line with previous studies ${ }^{33,43,52}$, we assume it to be prestressed (see figure 3a). The filaments are homogeneously prestretched by a factor $\xi>1$, which preserves the sixfold symmetry of the network $^{53}$. We can hence homogenize the discrete filament energy into an effectively isotropic continuous elastic membrane that we describe in the regime of finite elasticity (see SI p13-16). Its energy, expressed as a function of the incremental deformations from the prestressed state, is Hookean and includes a prestress term. Integrated over the membrane surface $A$, it reads $E=\int d A\left[\varepsilon_{i}+S_{\alpha \alpha}+M\left(\frac{1}{2}\left(e_{\alpha \beta}\right)^{2}+e_{\alpha \beta}^{2}\right)\right]$, where $\varepsilon_{i}$ is the constant energy density due to prestress and $e_{\alpha \beta}$ is the non-linear incremental membrane strain tensor. The prestressed network mechanics is characterized by an effective spectrin tension $S$ and an incremental shear modulus $M$, which are both functions of the prestretch ratio $\xi$, the phosphorylation activity $\left\langle n_{a}\right\rangle$ and the stress-free shear modulus $\mu_{0}$. For vanishing prestress $(\xi=1)$ the spectrin tension vanishes and $M=\langle\mu\rangle_{\text {(Supplementary figure S6). Extending a previous approach }}^{54}$, we derive the elastic forces of the spectrin network and expand them into vector spherical harmonics, which allows 
one to identify the three elementary modes of deformations: bending, stretching and shear. As usual in thin shell theory, bending and stretching deformations are linearly coupled for curved membranes, as illustrated in figure $3 \mathrm{c}$ for the harmonic $l=3$; by contrast, shear is decoupled here and is therefore ignored. The inextensible lipid bilayer is classically characterized by a bending modulus $\kappa$ and a surface tension $\sigma_{0}$, and its elastic forces can be derived from the CanhamHelfrich energy ${ }^{55,56} \mathrm{H}=\int\left[\kappa / 2 C^{2}+\sigma_{0}\right] d A$, where $C$ measure the mean local curvature.

The dissipative forces on the membrane have several sources. The inner and outer fluids have different viscosities and exert viscous stresses ${ }^{54}$, while the relative movement of the spectrin with respect to the lipid bilayer leads to a tangential friction force. Surprisingly this friction force is found to largely overcome viscous damping for large wavelengths; it therefore controls the tangential relaxation dynamics (Supplementary figure S6). A Langevin membrane dynamics is obtained by combining elastic and dissipative forces with thermal noise. This leads, for a given spherical harmonic $(l, m)$, to an effective dynamics coupling bending $u_{l m}^{(r)}$ and stretching $u_{l m}^{(1)}$ deformations

$\frac{d \mathbf{U}_{l m}(t)}{d t}=-\underline{\underline{\mathbf{W}}} \cdot \mathbf{U}_{l m}(t)+\mathbf{Z}_{l m}^{T H}(t)+\mathbf{Z}_{l m}^{A}(t)$

where $\underline{\underline{\mathbf{W}}}_{I m}$ is the relaxation frequency matrix for the membrane deformation $\mathbf{U}_{l m}=\left(u_{l m}^{(r)}, u_{l m}^{(1)}\right)^{\text {. }}$ The thermal noise $\mathbf{Z}_{l m}^{T H}(t)$ naturally contributes to both bending and stretching fluctuations, while metabolic shear modulus fluctuations $\mu_{l m}(t)$ generates, in an isolated network, direct active forces both in the tangential and normal directions. When the network is coupled to the bilayer, however, we predict the local bilayer incompressibility to systematically cancel the direct active normal force and we end up with an additional active source of noise for the stretching modes only, $\mathbf{Z}_{I m}^{A}(t)=\left(0, \Omega_{I m}^{A} \boldsymbol{u}_{l m}(t)\right)$. This is therefore in contrast with previous phenomenological active models based on the existence of a direct active normal force ${ }^{16,43}$. Tangential metabolic noise nevertheless perturbs normal membrane motions via the curvaturemediated coupling between bending and stretching modes (non-diagonal components of the matrix $\underline{\underline{\mathbf{W}}}_{I m}$ ). This effect is dampened by the friction between the bilayer and the network, and depends therefore critically on the tangentially fluid character of the bilayer. The membrane shape fluctuation spectrum for each spherical harmonic $(l, m)$ can be calculated in Fourier space as a sum of the dissipative part of the response function ${ }_{\chi_{l m}^{\prime \prime}}^{\prime \prime}(f)$ and this active contribution 


$$
C_{l m}(f)=\frac{2 k_{B} T}{2 \pi f} \chi_{l m}^{\prime \prime}(f)+\frac{2\left\langle n_{a}\right\rangle\left(1-\left\langle n_{a}\right\rangle\right) \tau_{a}}{1+\left(2 \pi f \tau_{a}\right)} \mid \mathrm{N}_{l m}(f)^{2}
$$

where $\mathrm{N}_{l m}(f)$ captures the complex mode- and frequency-dependent propagation of tangential active noise into membrane shape fluctuations. The deviation from FDT is characterized by an active timescale $\tau_{\mathrm{a}}=\left(k_{\mathrm{a}}+k_{\mathrm{i}}\right)^{-1}$, which controls the frequency, below which a departure from equilibrium is observed for a specific mode $(l, m)$ : for active timescales much lower than the typical membrane relaxation frequency $\left|\underline{\underline{\mathbf{W}}}_{I m}\right|$, the membrane does not have time to move and the metabolic activity is ineffective, whereas for larger active timescales the FDT violation increases and finally saturates at a value set by the maximum energy input in the system (see Supplementary figure 6). We calculate the fluctuation spectrum $C(f)$ as a sum over the relevant modes $(I \geq 2,-I \leq m \leq l)$ and predict a violation of FDT for non-zero metabolic activity $\left\langle n_{a}\right\rangle\left(1-\left\langle n_{a}\right\rangle\right)>0$ and for a prestressed spectrin network $\xi>1 \Leftrightarrow S>0$, in agreement with previous hypotheses ${ }^{43,44}$. In contrast, we find here that the violation vanishes for flat membranes $(R \rightarrow \infty)$. The membrane curvature is therefore a key feature for propagating tangential spectrin activity into normal shape motions, and we predict an increase of active fluctuations with membrane curvature, which may explain the higher fluctuations recently measured along the rim of the RBC, compared to its central region ${ }^{15,24}$. To fit the experimental data for rheological tests, we use typical mechanical parameters for the RBC, that have been either experimentally measured or estimated in simulations, and four fitting parameters (see Supplementary Table): the active rates $k_{a} \approx 1.12 s^{-1}$ and $k_{i} \approx 5.6 s^{-1}$, leading to a mean fraction of active sites $\left\langle n_{a}\right\rangle \approx 17 \%$, the network prestretch $\xi \approx 1.04$, controlling both passive and active spectrin mechanics, and the lipid bilayer excess area $\Delta \approx 1.9 \%$ which determines the bilayer tension ${ }^{57,58}$ (see SI p29-31). Our model shows excellent agreement with the passive response of the membrane and can reproduce the active fluctuation spectrum (figure 3d). A broader distribution of active timescales may refine this agreement and could arise from a Bell-like dependence of the transition rates on membrane extension $^{59}$. Interestingly, we find a slightly negative lipid bilayer tension $\sigma_{0} \approx-6.510^{-7} \mathrm{~J} \mathrm{~m}^{-2}$, which reveals a tangential compression of the bilayer, in agreement with a previous independent estimation $^{49}$. Writing the force balance for the harmonic $I=0$, which reduces to the Laplace's law $\Delta P=\frac{2\left(S+\sigma_{0}\right)}{R}$, we find that the elastic contribution arising from compression of the bilayer 
is large enough to counterbalance intrinsically the spectrin tension $S>0$ in the membrane, even in the absence of pressure jump (figure $3 b$ ). Our analytical model is therefore self-consistent and shows that the composite nature of the RBC membrane allows it to maintain an intrinsic prestress, a necessary feature for the emergence of spectrin-based active fluctuations.

To investigate the role of other potential metabolic processes in flickering, such as ion-pumps ${ }^{60}$ or phospholipid translocase ${ }^{61}$ activities, we employ a cell-scale in-silico model, which allows us to closely mimic the experimental situation, including the biconcave RBC geometry, the membrane curvature and stretching elasticity, the attached beads, and the optical tweezers. In our simulations, the hydrodynamics of the internal and external fluids and thermal fluctuations are taken into account by a particle-based mesoscale simulation technique, the dissipative particle dynamics method ${ }^{62,63}$. Our RBC model (figure $4 a$ ) is based on a two-dimensional triangulation of the composite membrane ${ }^{64}$, which corresponds to a network of viscoelastic springs with the inclusion of membrane bending energy and constraints for the conservation of membrane area and volume (see Supplementary Note, Section 1). The spring elements mimic the stretching elasticity of the spectrin network, and simultaneously capture viscous dissipation within the membrane. The bending energy describes bending resistance of the lipid bilayer, while the area and volume conservation constraints mimic the incompressibility of the lipid bilayer and cytosol, respectively. Modeling the RBC membrane at the spectrin level is still very challenging for realistic physical conditions (i.e., appropriate ambient temperature, membrane elasticity, and long times). To obtain an accurate averaging of fluctuations at the time scales of several minutes, which is much longer than a typical RBC relaxation time, we need to employ a coarse-grained model of the membrane, restricting its triangulation to about a thousand vertices. This coarsegrained description for a $\mathrm{RBC}$ has proved to reproduce quantitatively and accurately the viscoelasticity of the RBC membrane as well as deformation of RBCs due external forces (laser tweezers) and under flow ${ }^{64,65}$.

To simulate active processes, we furthermore add random active forces acting normally on membrane vertices (figure 4c). For ion pumps or other membrane protein activity the force exerted by an active center has to be counteracted by an exact opposite force in the surrounding fluid. The force density field of active centers have therefore a zero monopole moment, and the first contribution expected is a force dipole ${ }^{66}$.

As in experiments, the three handle beads in simulations are held by a harmonic potential mimicking optical tweezers, while the probe bead is moved sinusoidally to determine the mechanical response function (figure 4a-b). The free fluctuations of the probe bead are measured in a separate simulation. The simulations reproduce accurately the experimental response function (figure 4d), and lead to the estimation of mechanical properties of RBCs (Supplementary Table). The found mechanical properties are fully consistent with previous experimental estimations. Simulations reproduce the experimental violation of the FDT very precisely with the force amplitude $F_{0} \approx 650 \mathrm{fN}$ for active dipoles and with the transition rates $k_{a} \approx 0.42 \mathrm{~s}^{-1}$ and $k_{i} \approx 1.3 \mathrm{~s}^{-1}$ between active and inactive states (figure $4 \mathrm{c}$ ). The order of 
magnitude of the dipole force amplitude is consistent with simple estimations proposed for ionpumps ${ }^{67}$. The timescales are larger than those predicted analytically with the spectrin activity hypothesis, a difference, which may be explained by the large bilayer friction, dampening the propagation of tangential spectrin activity into normal membrane shape fluctuations. When active processes are turned off, the simulated membrane fluctuations correspond to the FDT predictions (figure $4 \mathrm{c}$, dashed lines) as expected. The resulting stochastic process for the membrane activity leads to an average fraction of active sites (i.e. those with the force applied at any moment of time) on a RBC of about $12 \%$. Thus, our RBC simulations show that simple uncorrelated active force dipoles can reproduce experimental results with excellent accuracy. The agreement between experiments and simulation result, using force dipoles, indicates that the spectrin network, as suggested by our and other theoretical models, may not be the only source of activity in the RBC membrane. In particular, the activity of ion pumps in the membrane might offer a compelling explanation for the active flickering we measure here directly ${ }^{60,67}$. Simulations also allow efficient testing of hypothetical active processes, such as local force monopoles, that may conserve momentum only globally. Such active force centers fit the data equally well with the same active rates as force dipoles but with ten fold lower force amplitudes $F_{0} \approx 50 \mathrm{fN}$, in agreement with previous estimations ${ }^{44}$ (Supplementary figure S7). Therefore, our simulations show that several, non-excluding, underlying mechanisms of uncorrelated active forces may explain membrane activity and suggest that further experimental characterizations are required to clearly identify the molecular mechanisms involved in flickering. They provide clear kinetic and amplitude predictions to guide future experiments. 


\section{Figures}

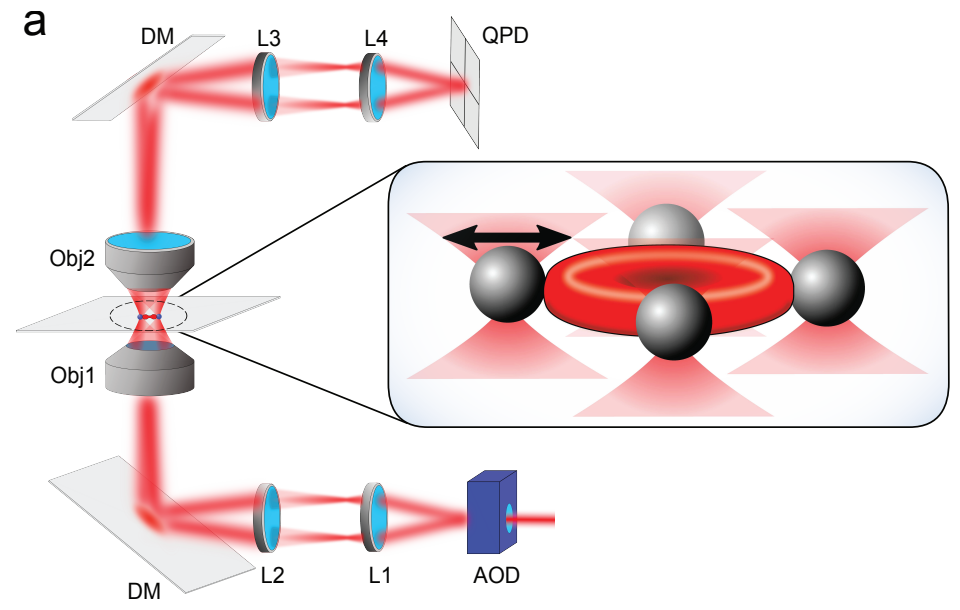

$\mathrm{b}$

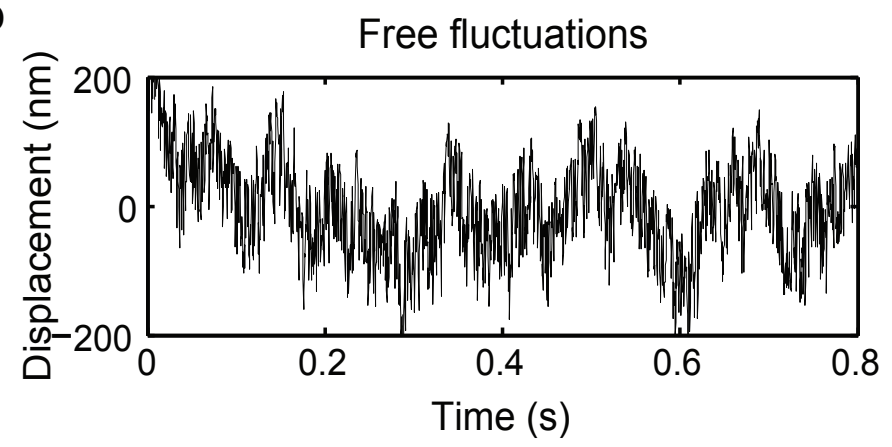

C Oscillating force and displacement at $19 \mathrm{~Hz}$

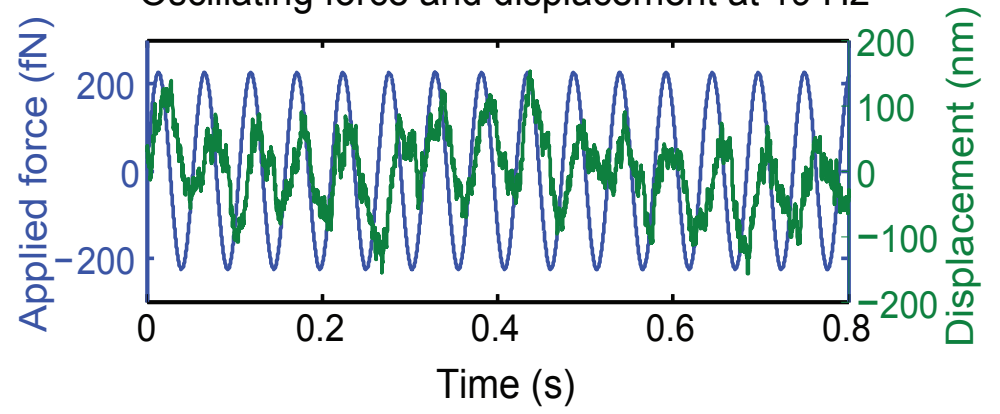

Figure 1: Experimental set-up. (a) The position of an IR laser is controlled by a pair of acoustooptical-deflectors (AOD) that allow rapid beam scanning ( $6 \mu$ s switching time). The beam is relayed by a telescope to enter the back focal plane of a $60 \mathrm{x} \mathrm{NA}=1.2$ water immersion objective (Obj1), and focused into the sample chamber. The light is collected using a 60x NA=0.9 water immersion objective (Obj2) and the back focal plane of this objective is imaged onto a quadrant photodiode (QPD). Four beads are attached to the RBC as sketched in the inset. One bead (marked by black arrow) is used as probe bead to either detect fluctuations or is moved by a sinusoidal force. (b) Typical time record of the membrane fluctuations. (c) Typical time record of the membrane response (green) to a sinusoidal force at $19 \mathrm{~Hz}$ (blue). The phase shift of about $20 \mathrm{~ms}$ is due to dissipation. 

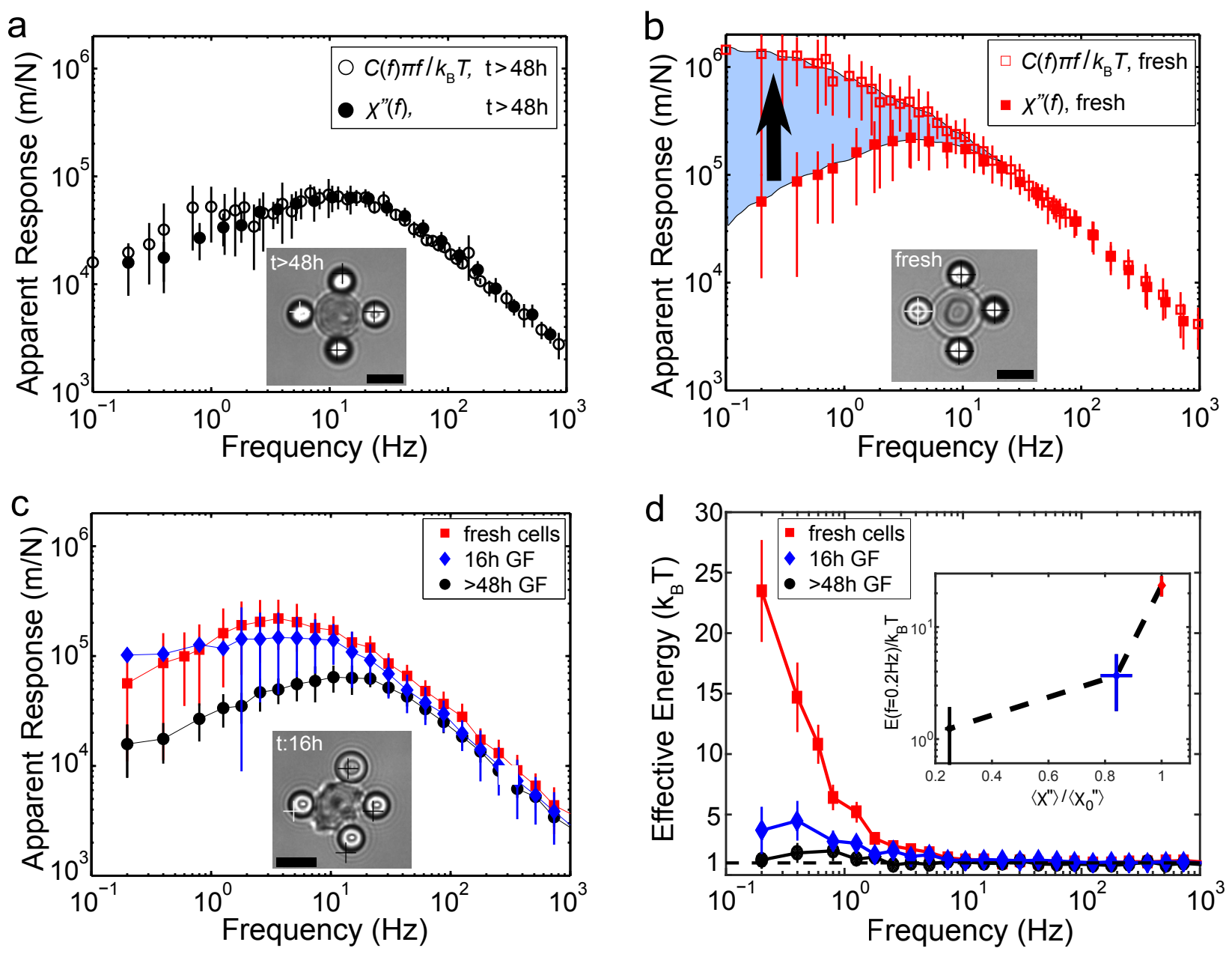

Figure 2: Membrane fluctuations and apparent response. Error bars denote s.d. (a) After 48h incubation in glucose free medium (GFM) at $37^{\circ} \mathrm{C}$, the measured response function (filled black circles) coincides with the response calculated from power spectral density $C(f)$ measurement (open black circles) using the fluctuation-dissipation theorem (Eq. 1). (Inset) RBC after $>48 \mathrm{~h}$ in GFM. (b) For fresh RBCs, the measured response (filled red squares) and the response calculated from fluctuations measurement (open red squares) differ below $10 \mathrm{~Hz}$ by more than an order of magnitude, indicating a clear violation of the FDT. (c) The dissipative response is moderately reduced for $16 \mathrm{~h}$ GFM, and strongly reduced for $>48 \mathrm{~h}$ GFM. (Inset) RBC after $>16 \mathrm{~h}$ in GFM. (d) Effective energy $E_{\text {eff }}(f)=C(f) \pi f / \chi^{\prime \prime}(f)$ for fresh RBCs (red), after 16h in GFM (blue) and for $>48 \mathrm{~h}$ in GFM (black). (Inset) Low frequency $(0.2 \mathrm{~Hz})$ effective energy as a function of relative mechanical changes. Scale bars are $5 \mu \mathrm{m}$. 

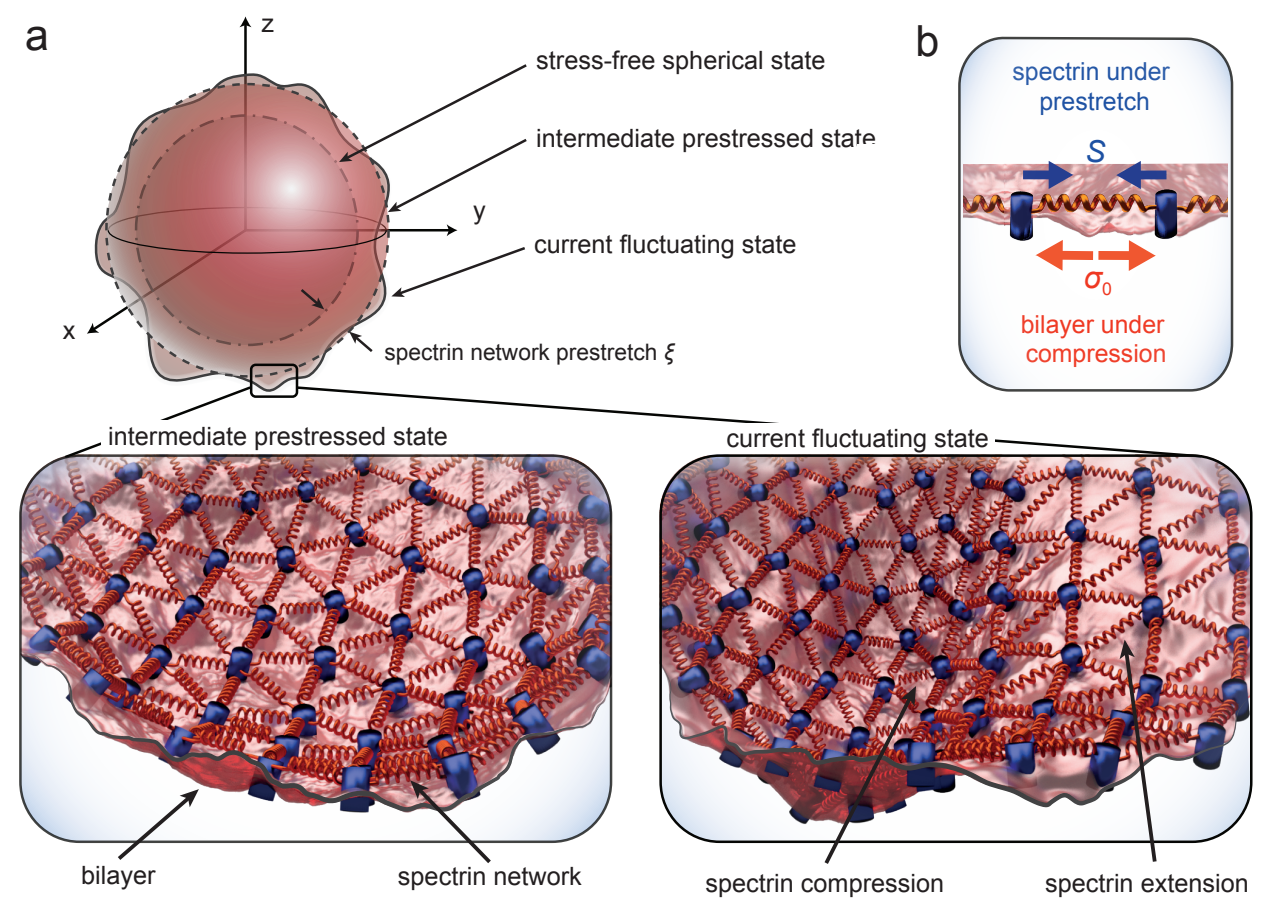

C

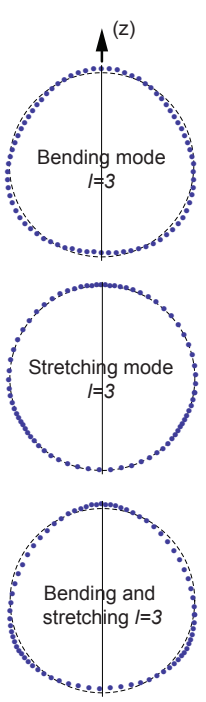

d

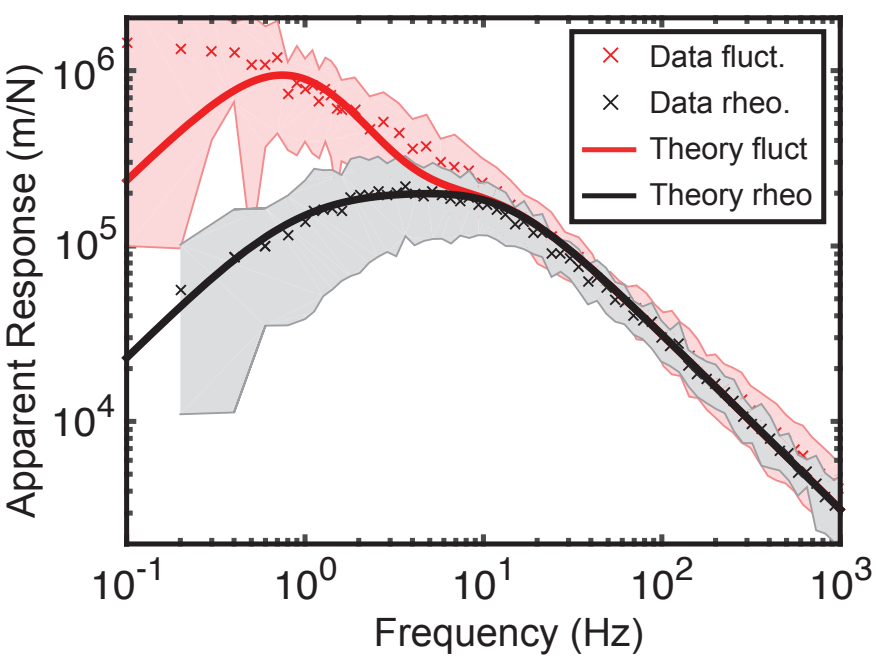

Figure 3: Analytic composite model of the red blood cell membrane. (a) Schematic description of the membrane geometry and configurations: the membrane is composed of the lipid bilayer and a regular triangular network of spring-like spectrin filaments. It fluctuates around an intermediate state where the spectrin network is prestressed and the bilayer compressed on average. (b) A large enough excess area of lipid bilayer creates a negative bilayer tension $\sigma_{0}$, as a result of its tangential compression. This bilayer elastic contribution can counterbalance the effective spectrin tension $S$, which results from filament prestretch. (c) The membrane deformation is a combination of bending and stretching modes, illustrated here for the spherical harmonic $l=3$. (d) The analytic model (plain lines) can reproduce the experimental fluctuations and response data (crosses). Shaded areas denote s.d. 

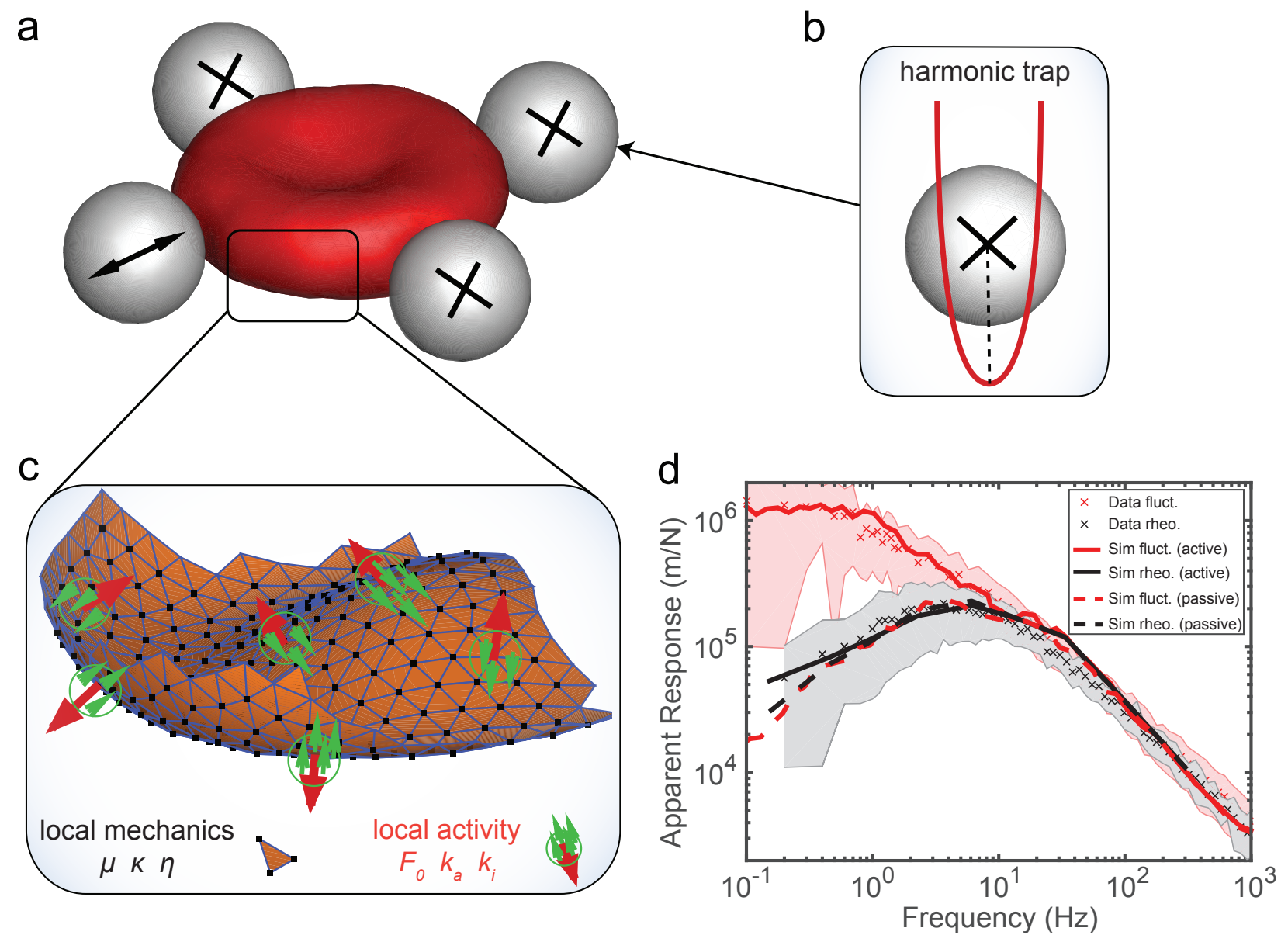

Figure 4: Simulations mimicking the experimental conditions. (a) Simulation setup with a RBC and four beads attached. Three beads (marked by crosses) are used as handles via a harmonic potential, while the fourth probe bead (marked by a two-side arrow) is sinusoidally driven to measure the mechanical response or let free for monitoring its fluctuations. (b) Illustration of a bead, whose center-of-mass is held in a harmonic potential. The harmonic potential mimics the optical tweezer trap in experiments and assumes the same strength for this trap. (c) A close-up of the membrane illustrating the coarse-grained membrane network structure and the forces applied to mimic activity of a RBC. The local membrane mechanics includes shear and bending moduli $\mu$ and $\kappa$, respectively, and surrounding fluids of viscosities $\eta$. The active forces at the membrane (drawn by red arrows) of amplitude $F_{0}$ are applied in the normal direction with equal probability inwards and outwards of the membrane. Their kinetics is characterized by active and inactive rates $k_{i}$ and $k_{a}$. The green arrows correspond to the counteracting forces applied locally to the surrounding fluids representing force dipoles. (d) Comparison of the simulation results with the experimental measurements. Simulations with force dipoles (active) reproduce well experimental measurement of the response (rheo.) and fluctuations (fluct.). When activity is turned off (passive), the FDT is recovered as in fully ATP-depleted RBCs (Figure 2a).

Shaded areas denote s.d. 


\section{Methods:}

Experimental preparation and effects on tension: Biotinylated red blood cells are prepared as described previously ${ }^{68}$ and mixed with streptavidin coated beads ( $3.28 \mu \mathrm{m}$ diameter). 4 beads are attached to the RBC in an opposing geometry (Figure 1) by trapping and manipulating the beads and the RBC using the multiplexed optical tweezers. The attachment area of the bead on the membrane was visually estimated to be about $1 \mu \mathrm{m}$ in diameter (Supplementary Methods, Supplementary Figure S2), which corresponds to about $2 \%$ of the RBC membrane. The resulting increase of tension can be estimated to be only about $0.2 \%$, and will hence not have a significant impact on the membrane fluctuations.

Bead and membrane motions: In the experiments we use the probe bead to determine the free fluctuations of the membrane, but also to apply a given force to the cell. The handle beads are exclusively used to keep the system in a stable 3D situation $20 \mu \mathrm{m}$ above the substrate. To test to which extend the probe bead fluctuations actually represent the membrane motion we also prepared sample cells with only three handle beads and measured the membrane fluctuations on the position where the fourth bead usually is attached using the methods reported previously ${ }^{6}$. Supplementary Figure S4 shows an overlay of the free probe-bead fluctuations and the free membrane. Within the error the two remain typically equal, and only at the low frequencies there is a systematic, but not significant difference between the probe-bead and the membrane. Interestingly, the membrane fluctuations in this 3D situation are a factor 5 higher than the fluctuations measured on the sedimented RBC (Supplementary Figure S4). Hence, when modeling the RBC membrane fluctuations, surface effects such as gravity mediated interactions as well as friction need to be taken into account for a correct analysis. To this end, our results can explain the previously found overestimation of RBC viscosities in the fluctuation analysis of sedimented cells ${ }^{6}$.

Effect of the trapping laser on measurements: To verify that the weak laser does not suppress any fluctuation, we compare the PSD of a free bead to the PSD of a bead attached to the RBC in the described experimental situation, using $0.1 \mathrm{~mW}$ in both cases. The resulting plot (Supplementary Figure S3) shows that the motion of the bead is indeed limited by the cell, as the free bead fluctuations are larger over the full frequency range.

Furthermore, we checked that the response function measured by the active microrheology does not simply reflect the trap stiffness of the handle beads. This was verified by comparing the PSD of the handle beads with the PSD derived from the response function measured (Supplementary Figure S8). The result shows that the response function of the cell is different to the response function imposed by the handle traps over the relevant frequency regime of $0.1-100 \mathrm{~Hz}$. Hence, the response function does indeed reflect the RBC mechanics.

Possible heating effects of the laser on the cell cannot be excluded. We can see hemolysis of cells if trapped with $100 \mathrm{~mW}$ for more than 30 minutes. However, with the applied laser intensities we do not observe any effect during the experiments. As both, the active and passive measurements 
are done in the same situation (handle beads trapped with $5 \mathrm{~mW}$ ), the typical heating should be similar in both situations and not lead to a systematic error of the measurements.

Finally, we can use the diffusive motion of the handle beads in the traps during the passive experiments to ensure that the determined PSD of the probe bead is not simply reflecting the fluctuations of the whole cell. Supplementary Figure S9 (a) shows the PSD of the probe bead and the handle beads in the passive experiments $(0.1 \mathrm{~mW}$ on the probe bead), while panel (b) gives the time-evolution of these motions. The fluctuations of the free probe bead are significantly larger which confirms that we can use it to infer information about the cell membrane fluctuations.

QPD and force calibration: For the correct measurement of both, the bead motion and the forces applied to the bead, the calibration of the QPD as well as the force calibration are key elements. These calibrations are done in situ on the beads attached to the cells. QPD calibration is obtained by a rapid scanning protocol that ensures no relevant bead motion during the scan (Supplementary Figure S10) and by an in situ force calibration (Supplementary Methods, Figure S9). As presented in Supplementary Figure S11, the presence of the cell does influence the position calibration by about $10 \%$. This also leads to a change in trap stiffness in the situation where the cell is attached to the beads (Supplementary Methods for details). Both effects are accounted for in the in situ calibration. It should be noted that the force calibration is possible even in the absence of a priori knowledge over the friction in the cell-bead system. The in situ calibration strategy becomes also important when the cells change their shape, for example under ATP depletion, where the used procedure ensures a correct interpretation of the measurements. As the QPD calibration depends on a precise interpretation of the QPD signal upon rapid laser motion, artifacts from the repositioning of the laser are suppressed by exclusively taking the data acquired during the time the laser rests at a given position (Supplementary Figure S12).

More details about the scanning routines, force calibration and the experimental procedure can be found in the Supplementary Methods. 


\section{Acknowledgments}

We thank V.L. Lorman and J. Prost for careful reading of the analytical model. T.B. was founded by ANR-11-JSV5-0002 and by the Deutsche Forschungsgemeinschaft (DFG), Cells-in-Motion Cluster of Excellence (EXC 1003 - CiM), University of Münster, Germany. H.T. acknowledges support from the Young Researcher Prize of the Bettencourt-Schueller Foundation. D.A.F. acknowledges funding by the Alexander von Humboldt Foundation. D.A.F., T.A., and G.G. gratefully acknowledge a CPU time grant by the Jülich Supercomputing Center. N.S.G. acknowledges the Institut Curie's Mayent-Rothschild Visiting Professor fund and Labex CelTisPhyBio for their support during the stay at the Institut Curie. N.S.G. is the incumbent of the Lee and William Abramowitz Professorial Chair of Biophysics, and thanks ISF Grant 580/12 for support.

\section{Author contributions}

T.B. and C.S. designed experiments; T.B. performed experiments; H.T., B.A. and J.-F.J. derived the analytical model; D.A.F., T.A. and G.G. designed the simulation setup; D.A.F. performed simulations; T.B., H.T. and D.A.F. analyzed the data; all authors discussed and interpreted results; all authors wrote the manuscript.

\section{Additional information}

The authors declare no competing financial interests. Supplementary Information accompanies this paper on www.nature.com/naturephysics. Reprints and permissions information is available online at www.nature.com/reprints. Correspondence and requests for materials should be addressed to T.B.

\section{References and Notes}

1. Browicz, T. Further observation of motion phenomena on red blood cells in pathological states. Zbl Med Wiss 28, 625-627 (1890).

2. Evans, E. A. \& Parsegian, V. A. Thermal-mechanical fluctuations enhance repulsion between bimolecular layers. Proc. Natl. Acad. Sci. U. S. A. 83, 7132-7136 (1986).

3. Lin, L. C.-L. \& Brown, F. L. H. Dynamics of Pinned Membranes with Application to Protein Diffusion on the Surface of Red Blood Cells. Biophys. J. 86, 764-780 (2004).

4. Cojoc, D. et al. Toward fast malaria detection by secondary speckle sensing microscopy. Biomed. Opt. Express 3, 991-1005 (2012).

5. Strey, H., Peterson, M. \& Sackmann, E. Measurement of erythrocyte membrane elasticity by flicker eigenmode decomposition. Biophys. J. 69, 478-88 (1995).

6. Betz, T., Lenz, M., Joanny, J.-F. \& Sykes, C. ATP-dependent mechanics of red blood cells. Proc. Natl. Acad. Sci. 106, 15320-15325 (2009).

7. Yoon, Y.-Z. et al. Flickering Analysis of Erythrocyte Mechanical Properties: Dependence on Oxygenation Level, Cell Shape, and Hydration Level. Biophys. J. 97, 1606-1615 (2009).

8. Brochard, F. \& Lennon, J. F. Frequency spectrum of the flicker phenomenon in erythrocytes. J. Phys. 36, 10351047 (1975).

9. Evans, J., Gratzer, W., Mohandas, N., Parker, K. \& Sleep, J. Fluctuations of the red blood cell membrane: relation to mechanical properties and lack of ATP dependence. Biophys. J. 94, 4134-44 (2008). 
10. Yoon, Y. Z., Kotar, J., Brown, A. T. \& Cicuta, P. Red blood cell dynamics: from spontaneous fluctuations to non-linear response. Soft Matter 7, 2042-2051 (2011).

11. Boss, D. et al. Spatially-Resolved Eigenmode Decomposition of Red Blood Cells Membrane Fluctuations Questions the Role of ATP in Flickering. PLoS ONE 7, e40667 (2012).

12. Puckeridge, M., Chapman, B. E., Conigrave, A. D. \& Kuchel, P. W. Membrane flickering of the human erythrocyte: physical and chemical effectors. Eur. Biophys. J. EBJ 43, 169-177 (2014).

13. Blowers, R., Clarkson, E. M. \& Maizels, M. Flicker phenomenon in human erythrocytes. J. Physiol. 113, 228239 (1951).

14. Tuvia, S. et al. Cell membrane fluctuations are regulated by medium macroviscosity: evidence for a metabolic driving force. Proc. Natl. Acad. Sci. U. S. A. 94, 5045-9 (1997).

15. Park, Y. et al. Metabolic remodeling of the human red blood cell membrane. Proc. Natl. Acad. Sci. 107, 12891294 (2010).

16. Rodríguez-García, R. et al. Direct Cytoskeleton Forces Cause Membrane Softening in Red Blood Cells. Biophys. J. 108, 2794-2806 (2015).

17. Weed, R. I., LaCelle, P. L. \& Merrill, E. W. Metabolic dependence of red cell deformability. J. Clin. Invest. 48, 795-809 (1969).

18. Chabanel, A., Reinhart, W. \& Chien, S. Increased resistance to membrane deformation of shape-transformed human red blood cells. Blood 69, 739-743 (1987).

19. Discher, D. E., Mohandas, N. \& Evans, E. A. Molecular maps of red cell deformation: hidden elasticity and in situ connectivity. Science 266, 1032-1035 (1994).

20. Nakao, M., Nakao, T. \& Yamazoe, S. Adenosine Triphosphate and Maintenance of Shape of the Human Red Cells. Nature 187, 945-946 (1960).

21. Sheetz, M. P. On the mechanism of ATP-induced shape changes in human erythrocyte membranes. I. The role of the spectrin complex. J. Cell Biol. 73, 638-646 (1977).

22. Manno, S., Takakuwa, Y., Nagao, K. \& Mohandas, N. Modulation of Erythrocyte Membrane Mechanical Function by -Spectrin Phosphorylation and Dephosphorylation. J. Biol. Chem. 270, 5659-5665 (1995).

23. Manno, S., Takakuwa, Y. \& Mohandas, N. Modulation of Erythrocyte Membrane Mechanical Function by Protein 4.1 Phosphorylation. J. Biol. Chem. 280, 7581-7587 (2005).

24. Monzel, C. et al. Measuring fast stochastic displacements of bio-membranes with dynamic optical displacement spectroscopy. Nat. Commun. 6, 8162 (2015).

25. Van Kampen, N. G. in Fluctuation Phenomena in Solids (Academic Press, 1965).

26. Schmidt, D. et al. Signature of a Nonharmonic Potential as Revealed from a Consistent Shape and Fluctuation Analysis of an Adherent Membrane. Phys. Rev. X 4, 021023 (2014).

27. Ben-Isaac, E. et al. Effective Temperature of Red-Blood-Cell Membrane Fluctuations. Phys. Rev. Lett. 106, 238103 (2011).

28. Callen, H. B. \& Welton, T. A. Irreversibility and Generalized Noise. Phys. Rev. 83, 34-40 (1951).

29. Martin, P., Hudspeth, A. J. \& Julicher, F. Comparison of a hair bundle's spontaneous oscillations with its response to mechanical stimulation reveals the underlying active process. Proc. Natl. Acad. Sci. 98, 1438014385 (2001).

30. Mizuno, D., Tardin, C., Schmidt, C. F. \& MacKintosh, F. C. Nonequilibrium Mechanics of Active Cytoskeletal Networks. Science 315, 370-373 (2007).

31. Mizuno, D., Bacabac, R., Tardin, C., Head, D. \& Schmidt, C. F. High-resolution probing of cellular force transmission. Phys. Rev. Lett. 102, 168102 (2009).

32. Hoffman, B. D., Massiera, G., Van Citters, K. M. \& Crocker, J. C. The consensus mechanics of cultured mammalian cells. Proc. Natl. Acad. Sci. 103, 10259-10264 (2006).

33. Discher, D. E., Boal, D. H. \& Boey, S. K. Simulations of the erythrocyte cytoskeleton at large deformation. II. Micropipette aspiration. Biophys. J. 75, 1584-1597 (1998).

34. Engelhardt, H., Gaub, H. \& Sackmann, E. Viscoelastic properties of erythrocyte membranes in high-frequency electric fields. Nature 307, 378-380 (1984).

35. Lenormand, G., Hénon, S., Richert, A., Siméon, J. \& Gallet, F. Direct Measurement of the Area Expansion and Shear Moduli of the Human Red Blood Cell Membrane Skeleton. Biophys. J. 81, 43-56 (2001).

36. Betz, T. \& Sykes, C. Time resolved membrane fluctuation spectroscopy. Soft Matter 8, 5317 (2012).

37. Chu, H. et al. Identification of cytoskeletal elements enclosing the ATP pools that fuel human red blood cell membrane cation pumps. Proc. Natl. Acad. Sci. 109, 12794-12799 (2012).

38. Bandmann, U., Monti, M. \& Wadsö, I. Clinical Physiology: Microcalorimetric Measurements of Heat Production in Whole Blood and Blood Cells of Normal Persons. Scand. J. Clin. Lab. Invest. 35, 121-127 (1975). 
39. Subrahmanyam, G., Bertics, P. J. \& Anderson, R. A. Phosphorylation of protein 4.1 on tyrosine-418 modulates its function in vitro. Proc. Natl. Acad. Sci. U. S. A. 88, 5222-5226 (1991).

40. Gauthier, E., Guo, X., Mohandas, N. \& An, X. Phosphorylation-Dependent Perturbations of the 4.1RAssociated Multiprotein Complex of the Erythrocyte Membrane. Biochemistry (Mosc.) 50, 4561-4567 (2011).

41. Lu, P. W., Soong, C. J. \& Tao, M. Phosphorylation of ankyrin decreases its affinity for spectrin tetramer. J. Biol. Chem. 260, 14958-14964 (1985).

42. Manno, S., Takakuwa, Y., Nagao, K. \& Mohandas, N. Modulation of erythrocyte membrane mechanical function by beta-spectrin phosphorylation and dephosphorylation. J. Biol. Chem. 270, 5659-5665 (1995).

43. Gov, N. S. \& Safran, S. A. Red Blood Cell Membrane Fluctuations and Shape Controlled by ATP-Induced Cytoskeletal Defects. Biophys. J. 88, 1859-1874 (2005).

44. Gov, N. S. Active elastic network: Cytoskeleton of the red blood cell. Phys. Rev. E 75, 011921 (2007).

45. Boal, D. H., Seifert, U. \& Zilker, A. Dual network model for red blood cell membranes. Phys. Rev. Lett. 69, 3405-3408 (1992).

46. Peng, Z., Asaro, R. J. \& Zhu, Q. Multiscale modelling of erythrocytes in Stokes flow. J. Fluid Mech. 686, 299337 (2011).

47. Li, H. \& Lykotrafitis, G. Two-Component Coarse-Grained Molecular-Dynamics Model for the Human Erythrocyte Membrane. Biophys. J. 102, 75-84 (2012).

48. Peng, Z. et al. Lipid bilayer and cytoskeletal interactions in a red blood cell. Proc. Natl. Acad. Sci. 110, 1335613361 (2013).

49. Fournier, J.-B., Lacoste, D. \& Raphaël, E. Fluctuation spectrum of fluid membranes coupled to an elastic meshwork: jump of the effective surface tension at the mesh size. Phys. Rev. Lett. 92, 018102 (2004).

50. Dubus, C. \& Fournier, J.-B. A Gaussian model for the membrane of red blood cells with cytoskeletal defects. Europhys. Lett. EPL 75, 181-187 (2006).

51. Zhang, R. \& Brown, F. L. H. Cytoskeleton mediated effective elastic properties of model red blood cell membranes. J. Chem. Phys. 129, 065101 (2008).

52. Paulose, J., Vliegenthart, G. A., Gompper, G. \& Nelson, D. R. Fluctuating shells under pressure. Proc. Natl. Acad. Sci. 109, 19551-19556 (2012).

53. Kantor, Y. Entropic elasticity of tethered solids. Phys. Rev. A 39, 6582-6586 (1989).

54. Rochal, S. B., Lorman, V. L. \& Mennessier, G. Viscoelastic dynamics of spherical composite vesicles. Phys. Rev. E 71, 021905 (2005).

55. Canham, P. B. The minimum energy of bending as a possible explanation of the biconcave shape of the human red blood cell. J. Theor. Biol. 26, 61-81 (1970).

56. Milner, S. T. \& Safran, S. A. Dynamical fluctuations of droplet microemulsions and vesicles. Phys. Rev. A 36, 4371-4379 (1987).

57. Seifert, U. The concept of effective tension for fluctuating vesicles. Z Phys B Condens Matter 97, 299-309 (1995).

58. Loubet, B., Seifert, U. \& Lomholt, M. A. Effective tension and fluctuations in active membranes. Phys. Rev. E Stat. Nonlin. Soft Matter Phys. 85, 031913 (2012).

59. Bihr, T., Seifert, U. \& Smith, A.-S. Nucleation of Ligand-Receptor Domains in Membrane Adhesion. Phys. Rev. Lett. 109, 258101 (2012).

60. Prost, J. \& Bruinsma, R. Shape fluctuations of active membranes. Europhys. Lett. EPL 33, 321-326 (1996).

61. Graham, T. R. Flippases and vesicle-mediated protein transport. Trends Cell Biol. 14, 670-677 (2004).

62. Hoogerbrugge, P. J. \& Koelman, J. M. V. A. Simulating Microscopic Hydrodynamic Phenomena with Dissipative Particle Dynamics. Europhys. Lett. EPL 19, 155-160 (1992).

63. Español, P. \& Warren, P. Statistical Mechanics of Dissipative Particle Dynamics. Europhys. Lett. EPL 30, 191$196(1995)$.

64. Fedosov, D. A., Caswell, B. \& Karniadakis, G. E. A Multiscale Red Blood Cell Model with Accurate Mechanics, Rheology, and Dynamics. Biophys. J. 98, 2215-2225 (2010).

65. Fedosov, D. A., Noguchi, H. \& Gompper, G. Multiscale modeling of blood flow: from single cells to blood rheology. Biomech. Model. Mechanobiol. 13, 239-258 (2014).

66. Manneville, J.-B., Bassereau, P., Ramaswamy, S. \& Prost, J. Active membrane fluctuations studied by micropipet aspiration. Phys. Rev. E 64, 021908 (2001).

67. Manneville, J.-B., Bassereau, P., Ramaswamy, S. \& Prost, J. Active membrane fluctuations studied by micropipet aspiration. Phys. Rev. E 64, (2001).

68. Pierrat, S., Brochard-Wyart, F. \& Nassoy, P. Enforced Detachment of Red Blood Cells Adhering to Surfaces: Statics and Dynamics. Biophys. J. 87, 2855-2869 (2004). 